\title{
老化对猕猴中颞视区细胞早期方向选择性的影响
}

\author{
梁 振 $^{1}$, 陈月明 ${ }^{1,}{ }^{*}$, 孟 雪 $^{1}$, 王 奕 $^{1}$, 周宝琢 ${ }^{2}$, 谢莹堂 ${ }^{1}$, 何文胜 ${ }^{1}$ \\ (1. 安徽医科大学 生命科学学院生物医学工程系, 安徽 合肥 230032; \\ 2. 中国科学技术大学 生命科学学院, 安徽 合肥 230027)
}

\begin{abstract}
摘要: 中颞视区(middle temporal area、MT/V5)在视觉运动处理过程中起着重要作用。MT 区神经元对物体运 动方向具有强选择性，而这种细胞的方向选择性被认为是运动方向知觉的神经基础，且已有实验表明方向选择性 由于受到注意影响, 而在时间进程上分为 2 个阶段。该研究组先前的实验发现麻醉狝猴 (Rhesus macaque) MT 区 细胞的方向选择性发生了衰退，但该衰退是整个时间进程上平均的结果，并不能在时间进程上揭示其神经机制。 因此, 为了进一步探索运动方向感知能力下降的神经机制, 该实验采用单细胞技术在麻醉猕猴的 MT 区研究了在 正常老化过程中 MT 区细胞的早期方向选择性变化(early stage direction selectivity, esDB), 结果表明老年狝猴 MT 区细胞早期方向选择性显著降低, 具有强早期方向选择性的细胞显著减少。该结果进一步揭示了 MT 区细胞方向 选择性在早期发生的衰退可能介导了视觉运动感知能力的下降。
\end{abstract}

关键词: 早期方向选择性; 中䯅视区; 老化

中图分类号: Q436; Q429+2; Q421; Q424

文献标志码: A

文章编号：0254-5853-(2012)05-0498-05

\section{Aging affects early stage direction selectivity of MT cells in rhesus monkeys}

\author{
LIANG Zhen ${ }^{1}$, CHEN Yue-Ming ${ }^{1, *}$, MENG Xue ${ }^{1}$, WANG Yi ${ }^{1}$, ZHOU Bao-Zhuo $^{2}$, \\ XIE Ying-Ying ${ }^{1}$, HE Wen-Sheng ${ }^{1}$ \\ (1. Department of Bio-Medical Engineering, Anhui Medical University, Hefei Anhui 230032, China; 2. Vision Research Laboratory, \\ School of Life Science, University of Science and Technology of China, Hefei Anhui 230027, China)
}

\begin{abstract}
The middle temporal area (MT/V5) plays an important role in motion processing. Neurons in this area have a strongly selective response to the moving direction of objects and as such, the selectivity of MT neurons was proposed to be a neural mechanism for the perception of motion. Our previous studies have found degradation in direction selectivity of MT neurons in old monkeys, but this direction selectivity was calculated during the whole response time and the results were not able to uncover the mechanism of motion perception over a time course. Furthermore, experiments have found that direction selectivity was enhanced by attention at a later stage. Therefore, the response should be excluded in experiments with anesthesia. To further characterize the neural mechanism over a time course, we investigated the age-related changes of direction selectivity in the early stage by comparing the proportions of direction selective MT cells in old and young macaque monkeys using in vivo single-cell recording techniques. Our results show that the proportion of early-stage-direction-selective cells is lower in old monkeys than in young monkeys, and that the early stage direction bias (esDB) of old MT cells decreased relative to young MT cells. Furthermore, the proportion of MT cells having strong early stage direction selectivity in old monkeys was decreased. Accordingly, the functional degradation in the early stage of MT cells may mediate perceptual declines of old primates in visual motion tasks.
\end{abstract}

Key words: Early stage direction selectivity; Middle temporal area; Aging

精确感知物体运动方向的能力对于生存来说 至关重要。近年来, 许多心理物理学实验表明该能
力在人类的正常老化过程中发生了衰退(Habak \& Faubert, 2000; Norman et al, 2003; Snowden \&

收稿日期: 2012-06-11; 接受日期：2012-07-10

基金项目：国家自然科学基金面上项目(31171082); 安徽省高等学校省级自然科学研究项目(KJ2012A154)

*通信作者(Corresponding author), E-mail: ahchen123@foxmail.com 
Kavanagh, 2006; Tran et al, 1998; Trick \& Silverman, 1991; Willis \& Anderson, 2000; Wist et al, 2000), 但 这种衰退不能完全由眼视光和视网膜的变化来解 释(Ball \& Sekuler, 1986)。因此, Spear 提出假说认为 这种衰退来源于视觉中枢的功能性退化 (Spear, 1993; Spear et al, 1994)。

在过去几年里, 已有不少研究使用单细胞记录 技术来探讨方向知觉的衰老机制。对于 MT 区的研 究表明, 老年狝猴 MT 区细胞的方向选择性和对比 敏感度均出现衰退(Liang et al, 2010; Yang et al, 2009)。众所周知, 方向选择性细胞在运动处理过程 中起着非常重要的作用, 同时 top-down 的注意在时 间进程的晚期(视觉刺激 $300 \mathrm{~ms}$ 之后)对方向选择性 产生调制作用(Bergen \& Julesz, 1983; Cavanagh et al, 1990; Martinez-Trujillo \& Treue, 2004; Roelfsema et al, 2007; Treisman \& Gelade, 1980)。在麻醉麻瘰的单 细胞记录过程中, top-down 的注意调制由于麻醉作 用而大幅度削弱。为了进一步探索运动方向感知能 力下降的神经机制, 有必要在时间进程的早期研究 在正常老化过程中 MT 区细胞方向选择性的变化。

该实验通过比较 MT 区细胞的早期方向选择 性, 研究了老化对猕猴 MT 区细胞功能的影响, 以 进一步揭示 MT 区早期方向选择性在老化进程中的 作用。

\section{1 材料与方法}

分别在 3 只青年( $4 \sim 6$ 岁)和 4 只老年 $(23 \sim 31$ 岁)狝猴的 MT 区记录细胞反应。根据整个生命周期 计算(Tigges et al, 1988), >4 岁的狝猴为发育成熟的 成年猕猴，>20 岁的狝猴开始进入衰老阶段(猕猴年 龄 20 岁=人类年龄 60 岁)。实验前, 每只猴子均由 专业医生进行眼科检查, 以确保猴子没有明显的眼 视光和视网膜病变, 眼底血管、晶状体透明度和黄 斑均正常。所有实验相关操作均得到了中国科学技 术大学生命科学学院动物伦理审查委员会的许可, 操作过程参考了美国国立卫生研究院 $(\mathrm{NIH})$ 实验动 物饲养及使用指南的相关内容。采取的一切措施皆 以减少实验动物的痛苦及使用数量为目标。

手术前，使用盐酸氯胺酮 $(10 \mathrm{mg} / \mathrm{kg}$ ) 对猕猴进 行引导麻醉, 继而用含有 $3 \sim 5 \%$ 异氟烷(Halothane) 的氧化亚氮/医用氧混合气体 $\left(\mathrm{N}_{2} \mathrm{O}: \mathrm{O}_{2}=70: 30\right)$ 维持麻 醉状态。动物达到适当的麻醉深度后, 在无菌条件 下，进行静脉和气管插管手术。所有创口及压力点
均施用长效局部麻醉药物( $2 \%$ 盐酸利多卡因软膏)。 使用筒剑毒[d-tubocurarine, $0.4 \mathrm{mg} /(\mathrm{kg} \cdot \mathrm{h})$ ]和三典季 胺芬[gallamine triethiodiede, $7 \mathrm{mg} /(\mathrm{kg} \cdot \mathrm{h})$ ]糖混合溶 液 $(5 \%)$ 诱导并通过静脉插管对其进行持续麻痹。 $\mathrm{N}_{2} \mathrm{O}(75 \%)$ 和 $\mathrm{O}_{2}(25 \%)$ 和异氟烷 $(0.25 \% \sim 1 \%)$ 的混合 气持续供应维持麻醉。使用人工呼吸机, 调节潮气 量使呼出的 $\mathrm{CO}_{2}$ 维持在 $~ 4 \%$ 。使用电热毯等保温 装置, 使动物体温维持在 $38{ }^{\circ} \mathrm{C}$ 。整个实验过程中 持续监视心率及脑电以衡量麻醉深度。

当将猕猴置于生命维持系统后, 调整麻醉深度 使青、老年猕猴的生命体征维持一致, 用接触镜隔 离保护眼睛。在整个实验过程中, 反复测量确定视 盘和中央凹的位置, 猕猴的眼睛无明显病变。中题 视区定位在耳杆向后 $2 \mathrm{~mm}$, 旁开 $15 \mathrm{~mm}$, 初级视皮 层定位在耳杆后 $3 \mathrm{~mm}$, 旁开 $2 \mathrm{~mm}$ 。以相应的点为 圆心, 开一个直径为 $4 \mathrm{~mm}$ 的洞, 去除硬脑膜暴露 脑组织, 在电极进入脑组织后使用 $4 \%$ 琼脂凝胶 (agar)覆盖㐿骨开孔, 保护脑组织。细胞外单细胞 电信号通过玻璃微电极( 内充 $4 \mathrm{M} \mathrm{NaCl}$ ) 进行记录, 电极阻抗选择在 2 5 $\mathrm{M} \Omega$ 。中题视区细胞的离心度 主要分布于 $2 \sim 40^{\circ}$, 初级视皮层细胞的离心度主要 分布于 $2 \sim 15^{\circ}$ 。电极使用液压式微推进器 (David Kopf Instruments, Tojunga, California) 推进。

由计算机产生的刺激图形显示于放置在实验 动物眼球前 $57 \mathrm{~cm}$ 处的 CRT 显示器 $(1024 \times 768,100$ $\mathrm{Hz}, \mathrm{G} 220$, SONY, Japan)。实验状态下显示器的平均 亮度为 $38.7 \mathrm{~cd} / \mathrm{m}^{2}$, 在实验动物角膜附近的环境光 照强度 $0.1 \mathrm{lux}$ 。实验所用生成刺激图形程序由基 于 MATLAB ${ }^{\mathrm{TM}}$ (Mathworks, Natick, MA, USA)的心 理物理学工具包(Psychtoolbox)开发完成。CRT 显示 器的亮度非线性通过软件自动矫正。

首先测量细胞的最优空间频率和时间频率，在 最优刺激大小、时间频率和空间频率下，以运动的 正弦光栅和格栅为刺激图形，每个刺激小节(trial) 时间 (2.5 s) 包含 5 个周期 (cycle), 刺激图形的 Michaelson 对比度设置在 $100 \%$; 对于测试的每个 方位随机呈现 2 个刺激小节(即每个方位共有 10 个 周期的正弦光栅和格柱刺激); 在每两个刺激小节 之间插入一个 $4 \mathrm{~s}$ 的空白图形用于获取神经元的自 发发放并避免刺激适应的产生，呈现空白图形时显 示器全屏呈现平均亮度; 对神经元的优势眼在其最 优的刺激图形大小以及最优的空间频率下进行记 录; 使用一组运动方向不同的刺激(共包括 24 个不 
同的方向, $0 \sim 345^{\circ}$ 每 $15^{\circ}$ 获取一个值)在神经元的最 优刺激参数下对神经元的方位调谐曲线进行描绘, 每个刺激图形的方位与其运动方向垂直。

单个神经元放电通过细胞外单细胞放大器 (DAGAN, USA)进行放大, 然后一路信号接入示波 器, 另一路信号经过数据采集卡 (National Instruments, USA)模数转换后存入计算机进行后续 分析。实验数据采集及分析程序使用 Igor (WaveMetrics, USA)开发。

分析早期方向选择性时，首先分析细胞对不同 方向视觉刺激产生反应的刺激后 $300 \mathrm{~ms}$ 内的直方 图 (post-stimulus time histogram, PSTH, bin 宽取 10 $\mathrm{ms}$ ); 叠加平均相同方向的 10 个刺激的刺激后时间 直方图, 取其均值反应减去细胞自发发放值作为衡 量细胞对该刺激反应幅度的指标; 描绘该细胞的方 向调谐曲线并计算其早期方向选择性强度和最优 方向。

早期方向选择性计算方法和我们先前计算方 向选择性的方法相同(Liang et al, 2010), 现简述如 下: 将细胞在每个刺激方位下的反应值以矢量方式 进行加和, 然后用加和后的矢量值除以矢量模的和 即可得到矢量形式的方向选择性指数(Leventhal et al，1995)。矢量形式的选择性指数方向即代表神经 元的最优方向, 其模的大小(即方向选择性 esDB)表
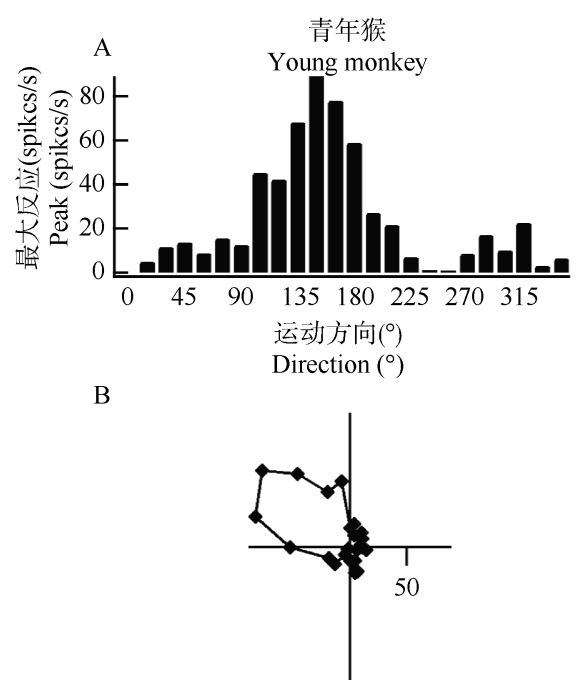

早期方向选择性 $=0.57$ $\mathrm{esDB}=0.57$
征细胞方向选择性的强弱。早期方向选择性指数 esDB 的取值范围为 $0 \sim 1$, esDB $=0$, 说明细胞完全 不具有方向选择性; $\mathrm{esDB}=1$, 说明细胞只对一个方 向的刺激产生反应; esDB $>0.2$ 表示该细胞有统计显 著意义的方向选择性强度 $(P<0.005$, Rayleigh 检验 $)$ 。

\section{2 实验结果}

分别在 3 只青年狝猴 MT 区记录到 84 个细胞, 4 只老年猕猴 MT 区记录到 98 个细胞，并测量细胞对 正弦光栅的反应, 且细胞的记录深度和离心度在 青、老年狝猴中一致。

绝大部分 MT 区神经元具有强方向选择性，且 在视觉信息运动处理过程中起着至关重要的作用 (Albright, 1984; Britten et al, 1992; Movshon et al, 1986; Newsome \& Pare, 1988)。图 1 为典型的青、老 年狝猴 MT 区神经元对正弦光栅的反应。从极坐标 图中可以看出老年猕猴的 MT 细胞调谐曲线显著性 宽于青年猕猴，即老年猕猴 MT 区细胞的方向选择 性降低。

青、老年猕猴 MT 区细胞早期方向选择性的详 细数据见表 1 。按照方向选择性强度将细胞分为无 选择性细胞 $(\mathrm{esDB}<0.1)$, 强选择性细胞 $(\mathrm{esDB}>0.2)$ 和极强选择性细胞 $(e s D B>0.4)$ 。结果表明老年猕猴 所具有的强选择性细胞 $(39.8 \%, 39 / 98)$ 相对青年猕
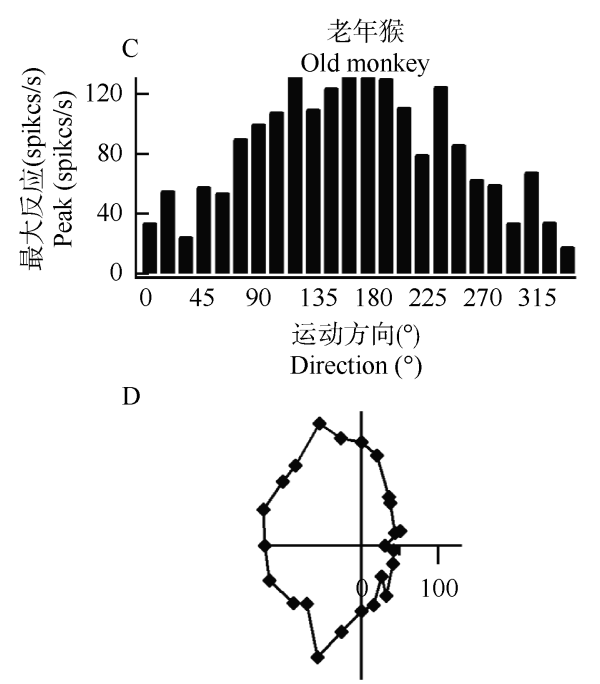

早期方向选择性 $=0.26$ $\mathrm{esDB}=0.26$

图 1 典型的青年狝猴和老年猕猴 MT 区神经元对正弦光栅的反应

Fig. 1 Tuning curves and corresponding polar plots obtained from young and old monkeys $\mathrm{A}, \mathrm{B})$ 青年猕猴; $\mathrm{C}, \mathrm{D})$ 老年猕猴。极坐标图上的点指示正弦光栅的运动方向，正弦光栅运动方向取自 $360^{\circ}$ 范围内等间隔 $\left(15^{\circ}\right)$ 的 24 个方向，且与 正弦光栅的方位垂直, 不同的运动方向随机呈现, 每个方向重复运动 10 次。

A, B) young monkeys; C, D) old monkeys. The stimuli were drifting sinusoidal gratings, randomly varied in 24 directions which ranged from $0^{\circ}$ to $360^{\circ}$ in $15^{\circ}$ steps. Each stimulus was presented in 10 cycles. Each point in the polar graph represents the response to the stimulus moving in the indicated direction. 
表 1 强选择性细胞和极强选择性细胞在青老年 MT 区 所占的比例

Tab. 1 Descriptive statistics of the proportions of MT cells between young and old groups

\begin{tabular}{ccc}
\hline & $\begin{array}{c}\text { 强选择性 } \\
\text { Strong directional bias } \\
(\text { esDB }>0.2)\end{array}$ & $\begin{array}{c}\text { 极强选择性 } \\
\text { Very strong directional bias } \\
(\text { esDB }>0.4)\end{array}$ \\
\hline Young & $72 / 84(85.7 \%)$ & $43 / 84(51.2 \%)$ \\
Old & $39 / 98(39.8 \%)$ & $10 / 98(10.2 \%)$ \\
$\begin{array}{c}\text { chi-square test } \\
P \text { value }\end{array}$ & $P \leqslant 0.001$ & $P \leqslant 0.001$ \\
\hline
\end{tabular}

使用列联表和卡方检验强选择性细胞和极强选择性细胞在青、老年 MT 区所占的比例, 强选择性细胞和极强选择性细胞所占的比例均显著减 少(chi-square test, $P \leqslant 0.01$ )。

The age-related changes in proportions of non-direction-biased cells (esDB $<0.1)$, strong-direction-biased cells (esDB $>0.4)$ and very-strongdirection-biased cells (esDB $>0.4$ ) were examined by using contingency tables. The proportions of strong-direction-biased and very-strong-directionbiased MT cells were decreased in old monkeys compared to those in young monkey groups.

猴( $85.7 \% ; 72 / 84$; chi-square test, $P \leqslant 0.001)$ 显著降低, 而极强选择性细胞受老化的影响最为严重, 从青年 狝猴的 $51.2 \%(43 / 84)$ 下降到老年猕猴的 $10.2 \%$ (10/98; $\chi 2$ test, $P \leqslant 0.001)$ 。

在该实验中, 我们还分析了 esDB 的分布(图 2), 84 个青年狝猴 MT 区细胞和 98 个老年猕猴 MT 区 细胞均参与了数据分析。老年狝猴 MT 区细胞的方 向选择性曲线左移, 表明老年猕猴 MT 区细胞的选 择性(mean $\pm S D, 0.18 \pm 0.12, n=98)$ 相比青年猕猴 MT

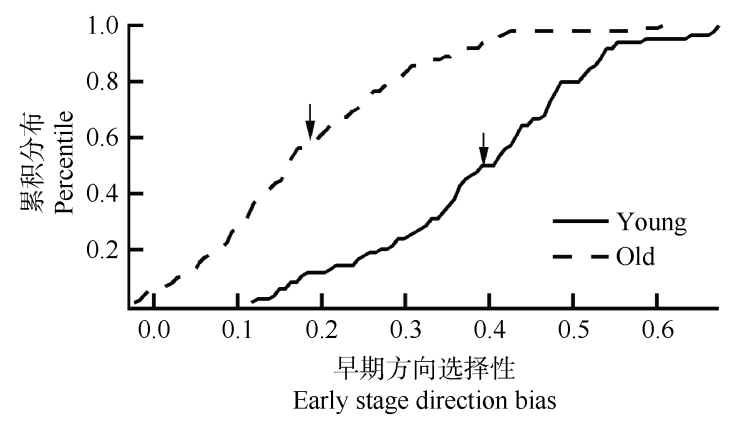

图 2 早期方向选择性的累积分布图

Fig. 2 Cumulative distribution of esDB in young and old monkey MT cells

青年猕猴 MT 区细胞共 84 个, 老年猕猴 MT 区细胞共 98 个; 实线和虚 线分别表示青年和老年猕猴 MT 区细胞的累积分布曲线; 箭头指向均值 所在的位置。

The percentage of young $(n=84)$ and old $(n=98)$ monkey MT cells with any given esDB is shown in cumulative distribution plots. Solid and dashed lines represent the data for young and old monkeys, respectively. The arrow on the line indicates the mean value. Old monkey cells showed decreased esDB values in comparison with young monkey cells.
区细胞 $($ mean $\pm S D: 0.39 \pm 0.13 ; n=84)$ 显著降低 $(t$-test, $P \leqslant 0.001$ )。

\section{3 讨 论}

本实验研究了老化对 MT 区早期方向选择性 的影响，结果表明与青年猕猴相比，老年猕猴的 MT 区细胞早期方向选择性显著降低。提示功能性 衰退发生在 MT 区, 且这种功能性衰退可能导致 了正常老化过程中视运动知觉的衰退。

众所周知, 方向选择性细胞在运动处理过程中 起着非常重要的作用，同时 top-down 的注意在时间 进程的晚期对方向选择性产生调制作用(Bergen \& Julesz, 1983; Cavanagh et al, 1990; Martinez-Trujillo \& Treue, 2004; Roelfsema et al, 2007; Treisman \& Gelade, 1980)。Liang et al (2010)发现老年猕猴 MT 区细胞方向选择性显著降低, 但这个衰退是整个时 间进程的平均结果, 并不能在时间进程上揭示其神 经机制以及老化在 down-top 和 top-down 过程中对 方向选择性的影响。本实验采用急性电生理方法, 在狝猴麻醉麻痹的状态下记录细胞反应。Top-down 的注意调制由于麻醉作用而大幅度削弱, 而 down-top 的方向选择性得到保留。因此, 在本实验 中我们选择在时间进程的早期研究在正常老化过 程中 MT 区细胞方向选择性的变化, 以揭示老化对 上行方向选择性的影响。该结果和我们先前的研究 结果一致。

目前, 我们发现与年龄相关的功能衰退可能是 由细胞次级结构的改变所导致的。因为先前的大量 实验表明猕猴和人类大脑皮层的细胞未发生丢失 (Morrison \& Hof, 1997, 2007; Peters et al, 1996, 1998; Wong, 2002; Yu et al, 2006), 特别是在 Superior Temporal Sulcus (STS) 区的实验亦未发现年龄相关 的细胞丢失(Duan et al, 2003; Morrison \& Hof, 2007)。MT 区位于 STS 区的后岸，同时向前额叶 46 区有大量投射，且 MT 区的神经元可以由在前额叶 46 区逆行染色而着色。前额叶 46 区逆行染色的神 经元树突棘的数量和密度显著降低将导致突触的 减少, 而突触是神经元功能的最小单位。因此, 这 种突触前、后的异常可能是导致 MT 区细胞功能性 衰退的原因。

神经化学递质的改变也可能导致老年视皮层 的衰退。我们的结果表明老年狝猴 MT 区细胞具有 高自发发放和诱发发放、低信噪比以及低方向选择 
性，表明皮层内的抑制回路可能发生了衰退。 Schmolesky et al (2000) 认为抑制回路的衰退可以 解释他们所发现的过度活动现象以及其他年龄相 关变化。而且，有实验发现在初级视皮层施加 GABA 及其激动剂可以抑制皮层细胞的过度活动, 并提高初级视皮层细胞的功能 (Leventhal et al, 2003)。近年来的心理物理学工作也表明老年人皮层 抑制功能降低(Betts et al, 2005; Butler \& Zacks, 2006)。另外, GABA 能中间神经元在 MT 区的广泛 分布(Thiele et al, 2004)也已被证明。综上所述, MT

\section{参考文献:}

Albright TD. 1984. Direction and orientation selectivity of neurons in visual area MT of the macaque [J]. J Neurophysiol, 52(6): 1106-1130.

Ball K, Sekuler R. 1986. Improving visual perception in older observers [J]. J Gerontol, 41(2): 176-182.

Bergen JR, Julesz B. 1983. Parallel versus serial processing in rapid pattern discrimination [J]. Nature, 303(5919): 696-698.

Betts LR, Taylor CP, Sekuler AB, Bennett PJ. 2005. Aging reduces center-surround antagonism in visual motion processing $[\mathrm{J}]$. Neuron, 45(3): 361-366.

Britten KH, Shadlen MN, Newsome WT, Movshon JA. 1992. The analysis of visual motion: a comparison of neuronal and psychophysical performance [J]. J Neurosci, 12(12): 4745-4765.

Butler KM, Zacks RT. 2006. Age deficits in the control of prepotent responses: evidence for an inhibitory decline [J]. Psychol Aging, 21(3): 638-643.

Cavanagh P, Arguin M, Treisman A. 1990. Effect of surface medium on visual search for orientation and size features [J]. J Exp Psychol Hum Percept Perform, 16(3): 479-491.

Duan H, Wearne SL, Rocher AB, Macedo A, Morrison JH, Hof PR. 2003. Age-related dendritic and spine changes in corticocortically projecting neurons in macaque monkeys [J]. Cereb Cortex, 13(9): 950-961.

Habak C, Faubert J. 2000. Larger effect of aging on the perception of higher-order stimuli [J]. Vision Res, 40(8): 943-950.

Leventhal AG, Thompson KG, Liu D, Zhou Y, Ault SJ. 1995. Concomitant sensitivity to orientation, direction, and color of cells in layers 2,3 , and 4 of monkey striate cortex [J]. J Neurosci, 15(3 Pt 1): 1808-1818.

Leventhal AG, Wang YC, Pu ML, Zhou YF, Ma YY. 2003. GABA and its agonists improved visual cortical function in senescent monkeys [J]. Science, 300(5620): 812-815

Liang Z, Yang Y, Li GX, Zhang J, Wang YC, Zhou YF, Leventhal AG. 2010. Aging affects the direction selectivity of MT cells in rhesus monkeys [J]. Neurobiol Aging, 31(5): 863-873.

Martinez-Trujillo JC, Treue S. 2004. Feature-based attention increases the selectivity of population responses in primate visual cortex $[\mathrm{J}]$. Curr Biol, 14(9): 744-751.

Morrison JH, Hof PR. 1997. Life and death of neurons in the aging brain [J]. Science, 278(5337): 412-419.

Morrison JH, Hof PR. 2007. Life and death of neurons in the aging cerebral cortex [J]. Int Rev Neurobiol, 81: 41-57.

Movshon JA, Adelson EH, Gizzi MS, Newsome WT. 1986. The analysis of moving visual patterns [J]. Exp Brain Res, 54(Suppl 2): 117-151.

Newsome WT, Pare EB. 1988. A selective impairment of motion perception following lesions of the middle temporal visual area (MT) $[\mathrm{J}] . J$ Neurosci, 8(6): 2201-2211.

Norman J F, Ross HE, Hawkes LM, Long JR. 2003. Aging and the perception of speed [J]. Perception, 32(1): 85-96.

Peters A, Morrison JH, Rosene DL, Hyman BT. 1998. Feature article: are
区细胞功能的衰退很可能由 GABA 能抑制系统的 退化所导致的。

老年人在运动敏感性和运动识别、鉴别上表现 衰退(Habak \& Faubert, 2000; Tran et al, 1998; Trick \& Silverman, 1991; Willis \& Anderson, 2000), 而这 些运动知觉相关的功能依赖于大脑中的方向选择 性细胞。我们发现的老年猕猴 MT 区细胞功能的衰 退可能导致了运动知觉的衰退, 即 MT 区细胞的功 能衰退可能是导致老年灵长类运动知觉退化的原 因之一。

neurons lost from the primate cerebral cortex during normal aging? [J] Cereb Cortex, 8(4): 295-300.

Peters A, Rosene DL, Moss MB, Kemper TL, Abraham CR, Tigges J, Albert MS. 1996. Neurobiological bases of age-related cognitive decline in the rhesus monkey [J]. J Neuropathol Exp Neurol, 55(8): 861-874.

Roelfsema PR, Tolboom M, Khayat PS. 2007. Different processing phases for features, figures, and selective attention in the primary visual cortex [J]. Neuron, 56(5): 785-792.

Schmolesky MT, Wang Y, Pu M, Leventhal AG., 2000. Degradation of stimulus selectivity of visual cortical cells in senescent rhesus monkeys [J]. Nat Neurosci, 3, 384-390.

Snowden RJ, Kavanagh E. 2006. Motion perception in the ageing visual system: minimum motion, motion coherence, and speed discrimination thresholds [J]. Perception, 35(1): 9-24.

Spear PD. 1993. Neural bases of visual deficits during aging [J]. Vision Res, 33(18): 2589-2609.

Spear PD, Moore RJ, Kim CB, Xue JT, Tumosa N. 1994. Effects of aging on the primate visual system: spatial and temporal processing by lateral geniculate neurons in young adult and old rhesus monkeys $[\mathrm{J}] . J$ Neurophysiol, 72(1): 402-420.

Thiele A, Distler C, Korbmacher H, Hoffmann KP. 2004. Contribution of inhibitory mechanisms to direction selectivity and response normalization in macaque middle temporal area [J]. Proc Natl Acad Sci USA, 101(26): 9810-9815.

Tigges J, Gordon TP, McClure HM, Hall EC, Peters A. 1988. Survival rate and life span of rhesus monkeys at the Yerkes regional primate research center [J]. Am J Primatol, 15(3): 263-273.

Tran DB, Silverman SE, Zimmerman K, Feldon SE. 1998. Age-related deterioration of motion perception and detection [J]. Graefes Arch Clin Exp Ophthalmol, 236(4): 269-273.

Treisman AM, Gelade G. 1980. A feature-integration theory of attention [J]. Cogn Psychol, 12(1): 97-136.

Trick GL, Silverman SE. 1991. Visual sensitivity to motion: age-related changes and deficits in senile dementia of the Alzheimer type [J]. Neurology, 41(9): 1437-1440.

Willis A, Anderson SJ. 2000. Effects of glaucoma and aging on photopic and scotopic motion perception [J]. Invest Ophthalmol Vis Sci, 41(1): 325-335.

Wist ER, Schrauf M, Ehrenstein WH. 2000. Dynamic vision based on motioncontrast: changes with age in adults [J]. Exp Brain Res, 134(3): 295-300.

Wong TP. 2002. Aging of the cerebral cortex [J]. McGill J Med, 6: 104-113.

Yang Y, Zhang J, Liang Z, Li GX, Wang YC, Ma YY, Zhou YF, Leventhal AG. 2009. Aging affects the neural representation of speed in Macaque area MT [J]. Cereb Cortex, 19(9): 1957-1967.

Yu S, Wang Y, Li X, Zhou Y, Leventhal AG. 2006. Functional degradation of extrastriate visual cortex in senescent rhesus monkeys $[\mathrm{J}]$. Neuroscience, 140(3): 1023-1029. 